\title{
Physical and Psychological Burden of Chronic Kidney Disease among Older Adults
}

\author{
William M. McClellan ${ }^{a, b} \quad$ Jerome Abramson ${ }^{a}$ Britt Newsome $^{b} \quad$ Ella Temple $^{c}$ \\ Virginia G. Wadley ${ }^{d}$ Paul Audhya ${ }^{f}$ Leslie A. McClure ${ }^{c}$ Virginia J. Howard ${ }^{e}$ \\ David G. Warnock ${ }^{\mathrm{b}}$ Paul Kimmel ${ }^{\mathrm{g}}$ \\ aDepartments of Medicine and Epidemiology, Rollins School of Public Health, Emory University, Atlanta, Ga., \\ ${ }^{b}$ Division of Nephrology, Department of Medicine, University of Alabama at Birmingham, ${ }^{C}$ Department of \\ Biostatistics, School of Public Health, University of Alabama at Birmingham, dDivision of Gerontology, \\ Geriatrics, and Palliative Care, Department of Medicine, University of Alabama at Birmingham, and \\ eDepartment of Epidemiology, School of Public Health, University of Alabama at Birmingham, Birmingham, Ala., \\ ${ }^{f}$ Amgen Corporation, Thousand Oaks, Calif., and 9Department of Medicine, George Washington University, \\ Washington, D.C., USA
}

\section{Key Words}

Functional status - Quality of life - Chronic kidney disease • End-stage renal disease $\cdot$ Glomerular filtration rate $\cdot$ REGARDS cohort study • Medical Outcomes Study Short Form-12

\begin{abstract}
Introduction: The purpose of the study is to determine if functional status and quality of life (QoL) vary with glomerular filtration rate (GFR) among older adults. Methods: We studied adults aged 45 years and older participating in the REasons for Geographic And Racial Differences in Stroke (REGARDS) cohort study. Data included demographic and health information, serum creatinine and hemoglobin, the 4-item Center for Epidemiologic Studies Depression Scale (CES-D-4), the 4-item Cohen's Perceived Stress Scale (PSS-4), reported health status and inactivity and the Medical Outcomes Study Short Form-12 (SF-12) QoL scores. Results: CKD (GFR $<60 \mathrm{ml} / \mathrm{min} / 1.73 \mathrm{~m}^{2}$ ) was present in $11.6 \%$ of the sub-
\end{abstract}

jects. As GFR declined, the SF-12 physical component score, adjusted for other participant attributes, declined from 38.9 to 35.9 ( $p=0.0001$ ). After adjustment for other risk factors, poorer personal health scores $(p<0.0001)$ and decreased physical activity $(p<0.0001)$ were reported as GFR declined. In contrast, after adjusting for other participant characteristics, depression scores and stress scores and the mental component score of the SF-12 were not associated with kidney function. Conclusion: Older individuals with CKD in the US population experience an increased prevalence of impaired QoL that cannot be fully explained by other individual characteristics.

Copyright $\odot 2010$ S. Karger AG, Basel

The views expressed in this article are those of the authors and as an unpublished document the contents may not be cited, quoted, or reproduced without the express permission of the authors.

\section{KARGER}

๑ 2010 S. Karger AG, Basel

Fax +41613061234 E-Mail karger@karger.ch www.karger.com www.karger.com/ajn
William McClellan, MD, MPH

Emory University, 1518 Clifton Road

Atlanta, GA 30322 (USA)

Tel. +1 404727 6976, Fax +1 4047278736

E-Mailwmcclel@sph.emory.edu 


\section{Introduction}

End-stage renal disease (ESRD) is associated with increased psychological distress and diminished quality of life (QoL) [1, 2]. Individuals with less severe chronic kidney disease (CKD) have also been reported to have impaired QoL [3-5]. Among patients with more severe CKD, QoL may be related to glomerular filtration rate (GFR) in a non-linear fashion. Participants in the Chronic Renal Insufficiency Cohort (CRIC) study had a decreased SF-36 physical component summary score as the GFR declined which was disproportionately reduced among individuals with a GFR $<30 \mathrm{ml} / \mathrm{min} / 1.73 \mathrm{~m}^{2}$ [6]. Similar patterns were seen for a CKD-specific measure of QoL, the Kidney Disease Quality of Life (KDQOL-36). The purpose of the current study is to describe the associations between individual measures of functional status, QoL measures, depression and perceived stress with decreasing GFR in a representative sample of the US population, and to test the prior hypotheses that the prevalence of these measures of disease burden varies with GFR in a non-linear fashion.

\section{Methods}

Study Design

REGARDS is a population-based cohort study of adults aged 45 years and older in the US population [7].

\section{Data}

Data collection methods have been published elsewhere [7] During a telephone interview, demographic information and selfreported comorbid conditions (hypertension, diabetes, heart failure, stroke and coronary heart disease, and cancer), cigarette smoking status, alcohol intake, education and income were obtained. A person's household income was classified as low if the reported income was less than USD 15,000.

During a subsequent in-home visit, height and weight were measured and blood specimens obtained for serum creatinine and hemoglobin. BMI was calculated as the weight in kilograms divided by the height in meters squared and anemia was defined as a hemoglobin value $<13.0 \mathrm{~g} / \mathrm{dl}$ for men and $<12.0 \mathrm{~g} / \mathrm{dl}$ for women.

\section{Depression}

The 4-item Center for Epidemiologic Studies Depression Scale (CES-D-4), derived from the original 20-item CESD, was used to measure depressive symptoms $[8,9]$. The CES-D-4 was developed to identify the presence of depressive symptoms and not as a diagnostic tool for clinical depression, and retains reliability and validity similar to the original 20 -item instrument [9]. The CESD-4 is a standard survey measure of depressive symptoms in older adults and has been previously used in patients with CKD [10]. The CES-D-4 uses a 4-point (0-3) scale to record specific symptoms of depression experienced during the preceding week and the scores range from 0 (no symptoms) to 12 . A CESD score of 4 was defined as increased prevalence of symptoms of depression $[10,11]$.

Stress

The short 4-item Cohen's Perceived Stress Scale (PSS-4) was used to assess perceived stress [12]. The PSS-4 consists of four questions: 'How often have you' (1) 'felt that you were unable to control the important things in your life', (2) 'felt confident about your ability to handle your personal problems', (3) 'found you could not cope with all the things that you had to do', and (4) 'felt difficulties were piling up so high that you could not overcome them'. Each item is scored on a 4-point scale from 'less than once a day' to '5-7 days' for a total possible score of 16 and scaled so that a low score was indicative of less stress. We defined a person as highly stressed if his or her score was $>7$. The PSS was not intended as a diagnostic tool and is used to compare stress levels among individuals within a specific sample [13].

\section{Perceived Poor Health}

Health status was ascertained by asking 'In general, would you say that your health is excellent, very good, good, fair or poor?' We defined the last category as poor health and for slope analyses we created an ordinal variable ranking from 1 for excellent health to 5 for poor health [14]. Self-reported health status has been shown to have a strong, independent association with subsequent changes in functional status, incident morbidity and mortality $[15,16]$.

\section{Inactivity}

Inactivity was ascertained by asking 'How would you compare your activity level to others your age? Would you say you are less active, same as others your age, more active, don't know/not sure?' We defined individuals as less active if they said yes to the first category.

\section{SF-12 QoL Score}

The Medical Outcomes Study Short Form-12 (SF-12) QoL scores are derived from 12 questions probing physical and mental well-being [14]. The SF-12 produces physical (PCS) and mental (MCS) component scores comparable to those from the full SF-36 questionnaire [14] and consists of questions on general health, vitality, bodily pain, social functioning, emotional and mental health, and physical functioning. The scores for both SF-12 scales range from 0 to 100 , with higher scores indicating better function. The instrument has been standardized with a population mean of 50 and an SD of 10 .

\section{Kidney Function}

Serum creatinine was measured by colorimetric reflectance spectrophotometry using the Ortho Vitros Clinical Chemistry System 950IRC instrument (Johnson \& Johnson Clinical Diagnostics, Rochester, N.Y., USA). In 2007 after completion of REGARDS recruitment, the REGARDS laboratory at the University of Vermont changed creatinine reagents to a method traceable to creatinine determined by isotope dilution mass spectrometry (IDMS). 50 samples were run in duplicate comparing the original method to the traceable method yielding the following calibration equation:

IDMS-traceable creatinine $=-0.06+0.953 \cdot$ creatinine. 
In addition, in 2007, 200 samples were sent from the REGARDS laboratory to the Cleveland Clinic for calibration resulting in the following calibration equation:

Calibrated creatinine $=-0.06+0.98 \cdot$ REGARDS creatinine.

As the two equations were nearly identical, the Vermont equation was used to convert original REGARDS creatinine values to IDMS-traceable values for determination of estimated GFR (eGFR), using the CKD-EPI estimating equation [17]:

$\mathrm{GFR}=141 \cdot \min (\mathrm{Scr} / \kappa, 1) \alpha \cdot \max (\mathrm{Scr} / \kappa, 1)^{-1.209}$.

$0.993^{\text {Age }} \cdot 1.018$ [if female] $\cdot 1.159$ [if Black],

where Scr is serum creatinine, $\kappa$ is 0.7 for females and 0.9 for males, $\alpha$ is -0.329 for females and -0.411 for males, min indicates the minimum of $S \mathrm{cr} / \mathrm{k}$ or 1 , and max indicates the maximum of Scr/К or 1 [17].

A single serum creatinine measurement was available for each participant. Individuals with a GFR $>89 \mathrm{ml} / \mathrm{min} / 1.73 \mathrm{~m}^{2}$ were collapsed into a single category. We used GFR $>89$ to $60 \mathrm{ml} /$ $\mathrm{min} / 1.73 \mathrm{~m}^{2}$ and we defined CKD as being present if the eGFR was between 15 and $59 \mathrm{ml} / \mathrm{min} / 1.73 \mathrm{~m}^{2}$.

\section{Statistical Analysis}

Means and proportions were used to describe the baseline characteristics and tests, ANOVA, and $\chi^{2}$ tests were used to test differences between groups as appropriate. Independent associations between outcomes and levels of kidney function were assessed using multivariable logistic and general linear models [18]. All of our multivariable models included age, gender, race, hypertension, diabetes mellitus, coronary heart disease, stroke, cancer, dyslipidemia, hemoglobin, and albumin level, BMI, income below poverty, education status, alcohol consumption, and smoking status as covariates.

We tested for linear trends in multivariable models by entering an ordinal variable for each of the functional or QoL attributes as the dependent variable and GFR as the linear predictor variable. We restricted these regression models to individuals with GFR $<60 \mathrm{ml} / \mathrm{min} / 1.73 \mathrm{~m}^{2}$. First we included both a linear and quadratic term for GFR to test for non-linearity; if the quadratic term was not significant $(p>0.05)$ we re-ran the model with only the first order, linear term for GFR. Analyses were conducted using SAS statistical software [19].

\section{Results}

There were 30,228 subjects recruited into the cohort at close of enrollment and 28,923 were included in our analyses. We excluded 1,305 participants: 1,191 missing serum creatinine and 114 with eGFR $<15$ as these subjects are defined as having ESRD and the QoL and psychological burden issues are different in this population. The mean (SD) was age of 65.3 (9.4) years; $59.2 \%$ were White and the remainder were Black and $45.3 \%$ were male. Current smoking was reported by $14.6 \%$ and $7.0 \%$ consumed more than one alcoholic beverage daily. Hypertension was present in $58.6 \%$, diabetes mellitus, $21.2 \%$, dyslipidemia, $58.6 \%$, coronary heart disease, $22.8 \%$, cerebrovascular disease, $9.9 \%$, and cancer, $14.5 \%$.

Nearly 13\% had less than a high school education, $25.8 \%$ completed high school, $45.9 \%$ had some post-high school education and $16.0 \%$ reported professional education. A low family income was reported by $11.0 \%$ of participants. The mean BMI was $29.3(6.2) \mathrm{kg} / \mathrm{m}^{2}$ and $1.0 \%$ of subjects were underweight and $38.1 \%$ were obese. The mean serum albumin level was $4.2(0.3) \mathrm{g} / \mathrm{dl}$ and $2.9 \%$ of participants had a serum albumin level $<3.5 \mathrm{~g} / \mathrm{dl}$. The mean hemoglobin was 13.7 (1.4) g/dl. CKD was present in $11.6 \%$ of the subjects. The mean (SD) GFR was 85.0 (19.8) $\mathrm{ml} / \mathrm{min} / 1.73 \mathrm{~m}^{2}$ with a $25-75$ th interquartile range from 73.0 to $98.2 \mathrm{ml} / \mathrm{min} / 1.73 \mathrm{~m}^{2}$.

All characteristics of subjects were associated with the level of kidney function (table 1). For example, mean age was 60.7 years among individuals with GFR $>90 \mathrm{ml} /$ $\min / 1.73 \mathrm{~m}^{2}$ and 70.8 years among those with a GFR of $15-29 \mathrm{ml} / \mathrm{min} / 1.73 \mathrm{~m}^{2}$. As the level of kidney disease became more severe the prevalence of cerebrovascular disease, coronary heart disease, hypertension, diabetes, cancer history, obesity, low albumin, anemia, less than high school education and low family income all tended to become more prevalent (table 1). In contrast, female gender, heavy alcohol use and current smoking all became less frequent. Finally, the prevalence of Black subjects was lower at earlier stages of kidney disease and higher at more severe stages (table 1).

The mean (SD) SF-12 MCS was 51.8 (7.6). As GFR declined from $\geq 90$ to $15-29 \mathrm{ml} / \mathrm{min} / 1.73 \mathrm{~m}^{2}$, the unadjusted MCS score increased slightly and then decreased among individuals with GFR $15-29 \mathrm{ml} / \mathrm{min} / 1.73 \mathrm{~m}^{2}$ (table 2). After adjusting for other participant characteristics, including age, gender, race, comorbidity (hypertension, diabetes mellitus, coronary heart disease, stroke, cancer, dyslipidemia, anemia, and albumin level), BMI, income below poverty, education status, alcohol consumption, and smoking status, neither the quadratic term for non-linearity $(\mathrm{p}=0.5024)$ nor the linear term ( $p=0.8137$ for the regression of the MCS score on GFR) were significant.

The mean (SD) SF-12 PCS score was 46.4 (10.4). The fully adjusted PCS declined from 40.9 to 37.3 as GFR declined from $\geq 90$ to $15-29 \mathrm{ml} / \mathrm{min} / 1.73 \mathrm{~m}^{2}$ (table 2). This relationship was non-linear, with both the highest and lowest GFR strata having lower PCS scores than the next adjacent strata, an inverted ' $U$ ' pattern. Both the linear $(\mathrm{p}<0.0001)$ and quadratic $(\mathrm{p}<0.0001)$ relationships with GFR were significant in the fully adjusted models. 
Table 1. Characteristics of REGARDS participants and associations between traits and level of kidney function

\begin{tabular}{|c|c|c|c|c|c|c|}
\hline & \multirow[t]{2}{*}{ All } & \multicolumn{5}{|c|}{$\mathrm{GFR}, \mathrm{ml} / \mathrm{min} / 1.73 \mathrm{~m}^{2}$} \\
\hline & & $>90$ & $60-89$ & $45-59$ & $30-44$ & $15-29$ \\
\hline \multirow[t]{2}{*}{ Age, mean $\pm \mathrm{SD}$} & $65.3 \pm 9.4$ & $60.7 \pm 7.7$ & $68.1 \pm 8.9$ & $72.4 \pm 8.7$ & $74.1 \pm 8.6$ & $70.8 \pm 9.5$ \\
\hline & $\mathrm{n}(\%)$ & \multicolumn{5}{|c|}{$\%$ subjects within GFR strata with trait } \\
\hline Female* & $15,819(54.7)$ & 58.6 & 50.7 & 55.3 & 56.5 & 48.7 \\
\hline Black* & $11,793(40.7)$ & 48.7 & 32.6 & 39.0 & 43.0 & 57.9 \\
\hline Smokes* & $4,195(14.6)$ & 18.8 & 11.3 & 9.95 & 11.9 & 12.4 \\
\hline Daily ETOH* & $1,974(6.7)$ & 7.5 & 7.1 & 4.9 & 2.8 & 5.1 \\
\hline $\mathrm{HBP}^{*}$ & $16,648(58.3)$ & 52.67 & 58.5 & 78.1 & 83.8 & 87.2 \\
\hline Diabetes* & $6,002(21.2)$ & 21.0 & 18.1 & 29.5 & 40.7 & 56.0 \\
\hline Dyslipidemia* & $16,798(58.6)$ & 53.8 & 60.4 & 69.2 & 72.9 & 74.2 \\
\hline Cerebrovascular disease ${ }^{*}$ & $2,849(9.9)$ & 7.2 & 10.0 & 18.4 & 23.1 & 25.5 \\
\hline Coronary heart disease ${ }^{*}$ & $6,424(22.8)$ & 17.5 & 24.6 & 34.3 & 37.5 & 47.2 \\
\hline History of cancer* & $2,517(14.5)$ & 10.8 & 16.2 & 20.6 & 23.7 & 16.4 \\
\hline Less than high school education* & $3,577(12.4)$ & 11.7 & 11.4 & 17.8 & 21.0 & 24.2 \\
\hline Low income* & $2,791(11.0)$ & 11.0 & 9.8 & 14.3 & 17.1 & 24.9 \\
\hline Obese ${ }^{*}$ & $10,964(38.1)$ & 41.7 & 34.2 & 38.6 & 40.0 & 43.4 \\
\hline Albumin $<3.5 \mathrm{~g}^{*}$ & $616(2.9)$ & 2.4 & 2.5 & 5.3 & 7.7 & 15.9 \\
\hline Hemoglobin, mean $\pm \mathrm{SD}^{*}$ & $13.7 \pm 1.4$ & $13.7 \pm 1.4$ & $13.8 \pm 1.4$ & $13.1 \pm 1.5$ & $12.6 \pm 1.6$ & $11.9 \pm 1.6$ \\
\hline
\end{tabular}

${ }^{*} \mathrm{p}<0.0001$ for equality of percents across levels of kidney function.

Table 2. Association between mean SF-12 scores and level of kidney function

\begin{tabular}{|c|c|c|c|c|}
\hline & \multicolumn{2}{|c|}{ SF-12 physical component scale score (PCS) } & \multicolumn{2}{|c|}{ SF-12 mental component scale score (MCS) } \\
\hline & crude & adjusted* & crude & adjusted ${ }^{*}$ \\
\hline \multicolumn{5}{|c|}{ GFR ml/min $/ 1.73 \mathrm{~m}^{2}$} \\
\hline$>90$ & 46.9 & 40.9 & 51.1 & 49.4 \\
\hline $60-89$ & 47.4 & 41.2 & 52.6 & 49.9 \\
\hline $45-59$ & 42.8 & 39.4 & 52.4 & 49.6 \\
\hline $30-44$ & 42.7 & 39.9 & 52.9 & 50.1 \\
\hline \multirow[t]{2}{*}{$15-29$} & 37.9 & 37.3 & 51.0 & 48.6 \\
\hline & $\mathrm{p}=0.0001$ & $\mathrm{p}=0.0001$ & $\mathrm{p}=0.1595$ & $\mathrm{p}=0.1600$ \\
\hline
\end{tabular}

* The mean score for each measure is reported as an unadjusted (crude) and fully adjusted score. We adjusted for characteristics included in table 1: age, gender, race, comorbidity (hypertension, diabetes mellitus, coronary heart disease, stroke, cancer, dyslipidemia, anemia, and albumin level), BMI, income below poverty, education status, alcohol consumption, and smoking status.

The mean (SD) CES-D-4 score of participants was 1.15 (2.1), and $7.6 \%$ of participants had a score of $\geq 4$, indicative of an elevated level of distress. The prevalence of elevated depressive symptoms increased from 6.4 to $12.7 \%$ as kidney function decreased from GFR $\geq 89-60$ to $15-$ $29 \mathrm{ml} / \mathrm{min} / 1.73 \mathrm{~m}^{2}(\mathrm{p}<0.0001)$. The likelihood of elevat- ed symptoms was increased twofold [OR $(95 \% \mathrm{CI})=2.13$ $(1.47,3.10)]$ among individuals with GFR $<30 \mathrm{ml} / \mathrm{min} / 1.73$ $\mathrm{m}^{2}$ compared to the referent group of GFR to $60-89 \mathrm{ml} /$ $\mathrm{min} / 1.73 \mathrm{~m}^{2}$, but this association did not persist after controlling for other participant characteristics [OR $(95 \%$ CI $)=1.35(0.62,2.96)]($ table 3$)$. 
Table 3. The odds ratio for the presence of depression and high stress level by level of kidney function

\begin{tabular}{|c|c|c|c|c|c|c|}
\hline & \multicolumn{3}{|c|}{ Depression } & \multicolumn{3}{|c|}{ Perceived stress } \\
\hline & $\%$ & crude & adjusted $^{*}$ & $\%$ & crude & adjusted ${ }^{*}$ \\
\hline \multicolumn{7}{|c|}{ GFR $\mathrm{ml} / \mathrm{min} / 1.73 \mathrm{~m}^{2}$} \\
\hline$>90$ & 8.8 & $1.42(1.29,1.56)$ & $1.23(0.99,1.52)$ & 16.6 & $1.43(1.34,1.54)$ & $1.13(0.96,1.33)$ \\
\hline $60-89$ & 6.4 & Ref. & Ref. & 12.2 & Ref. & Ref. \\
\hline $45-59$ & 7.0 & $1.10(0.92,1.31)$ & $0.82(0.54,1.25)$ & 14.1 & $1.19(1.04,1.36)$ & $1.01(0.74,1.36)$ \\
\hline $30-44$ & 7.5 & $1.19(0.91,1.56)$ & $0.75(0.39,1.44)$ & 16.5 & $1.43(1.18,1.73)$ & $0.66(0.40,1.11)$ \\
\hline $15-29$ & 12.4 & $2.13(1.47,3.10)$ & $1.35(0.62,2.96)$ & 22.4 & $2.08(1.55,2.81)$ & $1.23(0.64,2.36)$ \\
\hline
\end{tabular}

* These analyses used the CESD and PSS as categorical variables as described in Methods. We calculated the odds ratio (95\% CI) for the presence of depression and high stress compared to individuals with a GFR $\geq 60 \mathrm{ml} / \mathrm{min} / 1.73 \mathrm{~m}^{2}$. We report unadjusted (crude) comparisons and comparisons adjusted for charac- teristics included in table 1: age, gender, race, comorbidity (hypertension, diabetes mellitus, coronary heart disease, stroke, cancer, dyslipidemia, anemia, and albumin level), BMI, income below poverty, education status, alcohol consumption, and smoking status.

Table 4. Crude and adjusted QoL scores by level of kidney function

\begin{tabular}{|c|c|c|c|c|c|c|}
\hline & \multicolumn{2}{|l|}{ CESD } & \multicolumn{2}{|l|}{ PSS } & \multicolumn{2}{|c|}{ Perceived health } \\
\hline & crude & adjusted ${ }^{*}$ & crude & adjusted $^{*}$ & crude & adjusted* \\
\hline \multicolumn{7}{|c|}{ GFR $\mathrm{ml} / \mathrm{min} / 1.73 \mathrm{~m}^{2}$} \\
\hline$\geq 90$ & 1.29 & 2.18 & 3.30 & 4.20 & 2.62 & 3.30 \\
\hline $60-89$ & 1.0 & 2.00 & 2.86 & 4.09 & 2.46 & 3.23 \\
\hline $45-59$ & 1.08 & 1.90 & 3.14 & 4.15 & 2.93 & 3.44 \\
\hline $30-44$ & 1.00 & 1.76 & 2.99 & 3.79 & 3.04 & 3.48 \\
\hline \multirow[t]{2}{*}{$15-29$} & 1.71 & 2.18 & 3.79 & 4.16 & 3.53 & 3.71 \\
\hline & $\mathrm{p}<0.0001$ & $\mathrm{p}=0.035$ & $\mathrm{p}<0.0001$ & $\mathrm{p}=0.3062$ & $\mathrm{p}<0.0001$ & $\mathrm{p}<0.0001$ \\
\hline
\end{tabular}

* These analyses used the CESD, PSS and Perceived Health Scores as continuous variables. The mean score for each measure is reported as an unadjusted (crude) and fully adjusted score. We adjusted for characteristics included in table 1: age, gender, race, comorbidity (hypertension, diabetes mellitus, coronary heart disease, stroke, cancer, dyslipidemia, anemia, and albumin level), $\mathrm{BMI}$, income below poverty, education status, alcohol consumption, and smoking status.
The CES-D-4 depression score declined at lower levels of GFR until GFR of $15-29 \mathrm{ml} / \mathrm{min} / 1.73 \mathrm{~m}^{2}$ and then increased $(\mathrm{p}<0.0001)$ (table 4$)$. This relationship was significant $(\mathrm{p}=0.035)$ after adjusting for other covariates. Both the quadratic $(\mathrm{p}=0.2408)$ and linear $(\mathrm{p}=0.0647)$ slopes for the association between GFR and CESD-4 score were non-significant.

The mean (SD) PSS stress score was 3.2 (2.9), and $14.5 \%$ had a score of $\geq 7$ on the scale, indicative of a perception of high stress. The prevalence of high stress increased with decreasing GFR, rising from $12.2 \%$ among individuals with a GFR $60-89 \mathrm{ml} / \mathrm{min} / 1.73 \mathrm{~m}^{2}$ to $22.4 \%$ among individuals with a GFR of $15-29 \mathrm{ml} / \mathrm{min} / 1.73 \mathrm{~m}^{2}$ $[\mathrm{OR}(95 \% \mathrm{CI})=2.08(1.55,2.81)]$. This association, how- ever, did not persist after controlling for other participant characteristics [OR $(95 \% \mathrm{CI})=1.22(0.59,2.50)]$ (table 3). The adjusted mean stress score did not change as GFR declined (table 4) with quadratic $(\mathrm{p}=0.2797)$ and linear $(\mathrm{p}=0.1395)$ slopes that were not statistically significant.

The prevalence of reported poor health (score of 5) increased from 3.2 to $17.8 \%$ as GFR decreased from $\geq 90$ to $15-19 \mathrm{ml} / \mathrm{min} / 1.73 \mathrm{~m}^{2}(\mathrm{p}<0.0001)$. The odds of perceived poor health was over eightfold greater [OR $(95 \% \mathrm{CI})=$ $8.58(4.22,17.4)]$ among individuals with GFR $<30 \mathrm{ml} /$ $\mathrm{min} / 1.73 \mathrm{~m}^{2}$ compared to those with GFR $60-89 \mathrm{ml} /$ $\mathrm{min} / 1.73 \mathrm{~m}^{2}$ which declined [OR $(95 \% \mathrm{CI})=4.15(1.98$, 8.69)] after controlling for other participant characteristics (table 5). The mean (SD) perceived health score was 
Table 5. The odds ratio for the presence of poor perceived health and impaired activity level scores and kidney function

\begin{tabular}{|c|c|c|c|c|c|c|}
\hline & \multicolumn{3}{|c|}{ Perceived poor health } & \multicolumn{3}{|c|}{ Impaired activity } \\
\hline & $\%$ & crude & adjusted $^{*}$ & $\%$ & crude & adjusted ${ }^{*}$ \\
\hline \multicolumn{7}{|c|}{ GFR $\mathrm{ml} / \mathrm{min} / 1.73 \mathrm{~m}^{2}$} \\
\hline$\geq 90$ & 3.2 & $1.15(0.99,1.32)$ & $1.33(0.99,1.86)$ & 22.8 & $1.49(1.39,1.58)$ & $1.21(1.05,1.40)$ \\
\hline $60-89$ & 2.8 & Ref. & Ref. & 16.6 & Ref. & Ref. \\
\hline $45-59$ & 4.9 & $1.78(1.43,0.99)$ & $1.25(0.74,2.09)$ & 26.0 & $1.77(1.59,1.97)$ & $1.66(1.29,2.14)$ \\
\hline $30-44$ & 7.4 & $3.65(2.20,6.07)$ & $1.63(0.82,3.24)$ & 30.0 & $2.16(1.85,2.53)$ & $1.47(1.01,2.12)$ \\
\hline $15-29$ & 17.8 & $8.58(4.22,17.4)$ & $4.15(1.98,8.69)$ & 42.5 & $3.72(2.88,4.79)$ & $1.67(0.93,3.00)$ \\
\hline
\end{tabular}

* We calculated the odds ratio $(95 \% \mathrm{CI})$ for the presence of poor perceived health and impaired physical activity compared to individuals with a GFR $\geq 60 \mathrm{ml} / \mathrm{min} / 1.73 \mathrm{~m}^{2}$. We report unadjusted (crude) comparisons and comparisons adjusted for characteristics included in table 1: age, gender, race, comorbidity (hypertension, diabetes mellitus, coronary heart disease, stroke, cancer, dyslipidemia, anemia, and albumin level), BMI, income below poverty, education status, alcohol consumption, and smoking status.
2.6 (1.0), and 3.4\% had a score of 5 on the scale, indicative of a perception of poor health. The perceived health score, with higher scores indicative of a poorer perception of personal health, increased from 2.62 to 3.53 ( $\mathrm{p}<0.0001)$ as GFR declined from $\geq 90$ to $15-29 \mathrm{ml} / \mathrm{min} / 1.73 \mathrm{~m}^{2}$, and this increase, although substantially attenuated after adjustment to an increase from 3.30 to 3.71 , remained statistically significant $(\mathrm{p}=0.0001)$ (table 4$)$. Neither the fully adjusted quadratic slope $(\mathrm{p}=0.1653)$ nor the linear $(\mathrm{p}=0.1395)$ slope were significant.

The prevalence of inactivity was $20.6 \%$ and increased from $22.6 \%$ among individuals with a GFR $\geq 90 \mathrm{ml} /$ $\mathrm{min} / 1.73 \mathrm{~m}^{2}$ to $42.5 \%$ among those with GFR between 15 and $19 \mathrm{ml} / \mathrm{min} / 1.73 \mathrm{~m}^{2}(\mathrm{p}<0.0001)$. The odds of inactivity was nearly fourfold greater [OR $(95 \% \mathrm{CI})=3.72(2.88$, 4.79)] among individuals with GFR $<30 \mathrm{ml} / \mathrm{min} / 1.73 \mathrm{~m}^{2}$ compared to those with GFR $60-89 \mathrm{ml} / \mathrm{min} / 1.73 \mathrm{~m}^{2}$, which declined [OR $(95 \% \mathrm{CI})=1.67(0.78,2.86)]$ after controlling for other participant characteristics (table 5). Below $60 \mathrm{ml} / \mathrm{min} / 1.73 \mathrm{~m}^{2}$, the quadratic slope for impaired activity $(\beta=-0.0001, \mathrm{p}=0.0651)$ was marginally significant and the linear slope $(\beta=-0.0048, \mathrm{p}<0.0001)$ was significant.

\section{Discussion}

Our main findings are that, at lower levels of GFR, individuals in the US population report an increased burden of functional limitations and diminished QoL which is significantly attenuated, but not fully accounted for, by the presence of other participant characteristics. Al- though the diminished QoL and functional status we observed occurred mainly among participants with stage 4 CKD $\left(\mathrm{GFR}<30 \mathrm{ml} / \mathrm{min} / 1.73 \mathrm{~m}^{2}\right)$, we were unable to confirm a previously reported non-linear relationship for these factors with increasing severity of kidney disease. Information about the extent of diminished functional status and QoL and the level of kidney function at which limitation occurs is important to our understanding the burden of kidney disease in the estimated $8.5 \%$ of the US population with a GFR $<60 \mathrm{ml} / \mathrm{min} / 1.73 \mathrm{~m}^{2}$ [20].

Earlier studies have reported relationships between increasing severity of impaired kidney function and increased prevalence of decreased well-being and reported health, decreased physical function and increased prevalence of depression [6, 4, 21-24]. Our observations of an inverse relationship between activity level and GFR are consistent with estimates of functional impairment available from NHANES III where age-adjusted prevalence of inability to walk one-quarter mile increased from 6 to $15 \%$ and to lift $10 \mathrm{lb}$ from 6 to $15 \%$ as GFR among adult NHANES III participants decreased from 60 to $15 \mathrm{ml} /$ $\min / 1.73 \mathrm{~m}^{2}$ [25].

Chow et al. [26] examined SF-36 scores among over 10,000 participants in the Australian Diabetes, Obesity and Lifestyle Study. They found that, after adjusting for other participant characteristics, SF-36 Physical Functioning, Role-Physical, General Health, Vitality, and Role-Emotional scores were substantially lower among individuals with a GFR $<60 \mathrm{ml} / \mathrm{min} / 1.73 \mathrm{~m}^{2}$ compared to participants with higher GFR. Perlman et al. [27] made similar observations for a clinic-based population of individuals with stage 4 CKD. They found that SF-36 PCS 
scores, but not MCS scores, were substantially lower among their subjects compared to the general population. These results are consistent with other clinical trials or observational studies that failed to show meaningful diminution in the MCS scores in ESRD patients $[3,28-$ 33].

The association between prevalence of impaired functional status and QoL and GFR was linear and, with the exception of the SF-12 mental component score, quadratic terms in the fully adjusted models were not significant. The non-linear SF-12 MSC adjusted score was an invert ' $U$ ' pattern, where the score declined at both higher and lower GFR. We cannot, however, fully exclude non-linearity in these relationships and note that an apparent threshold for increased prevalence of CKD-related morbidity around $30 \mathrm{ml} / \mathrm{min} / 1.73 \mathrm{~m}^{2}$ has been reported for increased prevalence of anemia [34], acidosis and renal bone disease [35], mortality and progression to ESRD [36].

Our failure to find an association between decreasing GFR and depressive symptoms is inconsistent with a recent report by Hedayati et al. [37]. They used the Beck Depression Inventory (BDI) to screen heart failure patients for depression, followed by the National Institute of Mental Health Diagnostic Interview Schedule for individuals with BDI scores $>10$. The reported prevalence of depression was $22 \%$ in subjects with a GFR $<30 \mathrm{ml} /$ $\mathrm{min} / 1.73 \mathrm{~m}^{2}$ compared to $13 \%$ of those with less severe CKD. In contrast, Odden et al. [38] used the Patient Health Questionnaire (PHQ) to assess the presence of depression among participants in the Heart and Soul Study. They found that prevalence of depression did not vary between those with and those without CKD. Other observational studies of patients with advanced CKD often demonstrate that mean levels of BDI scores are not greatly above the cutoff for depression in the general population $[39,40]$.

It is interesting that patient perception of poor health status differed with levels of GFR. Higher scores on the Illness Effects Questionnaire, a global, burden of illness scale, were reported to be associated with mortality in ESRD patients in a previous study [41]. It is possible that an instrument designed to focus on perception of burden of illness and physical activity for moderate and advanced stage CKD patients might yield meaningful information for predicting outcome and monitoring interventions.

It might be argued that our results might be due, in part, to multiple comparisons that yielded statistically significant associations that occurred through chance alone. We feel that this is unlikely. First, our hypotheses were posited prior to inspection of the data, defining the scope of the multiple testing as the six health-related QoL measures we tested. If we adjust our nominal type $1 \alpha$ of 0.05 to accommodate each test, we have a nominal $\alpha$ of 0.008 , which is substantially greater than the level of the statistical support for the crude associations we noted, which was generally $\mathrm{p}<0.0001$. Further, the consistency of the associations noted argues for the non-random nature of the relationship between impaired kidney function and measures of functional status and QoL.

These observations have clinical implications for management of progressive kidney disease. The generally accepted measure of a clinically meaningful change in a health-related QoL score is one that is $\geq 50 \%$ of the score's standard deviation. In the general population, where the mean and standard deviation of the SF-12 are 50 and 10 , respectively, this translates to a difference of 5 units [42]. A second metric uses a standardized mean response (SMR), calculated as the mean difference divided by the $\mathrm{SD}$ of the mean, to assess the clinical importance of a HRQoL score change [43]. An SMR between 0.2 and 0.49 characterizes a small, $0.5-0.79$, a moderate, and 0.8 and above a large change in QoL.

Extrapolating from these change score metrics, the differences we found in the SF-12 PSC score with descending GFR were associated with corresponding modest, but clinically significant decrements in QoL. The fully adjusted PCS score ranged from 38.9 to 35 as GFR fell from $>60$ to $15 \mathrm{ml} / \mathrm{min} / 1.73 \mathrm{~m}^{2}$, yielding an SMR of 0.49 for the PCS score SD of 10, and the difference in the PCS score was $40 \%$ of the SD. The magnitude of these differences are comparable to those reported by Lim and Fisher [44] for changes in the PCS score for SF-12 Health Survey in Australian patients with acute heart disease and stroke.

Our results suggest that clinicians should explicitly inquire about decreased physical function and decreasing QoL when interviewing patients with advancing CKD. Clinicians should be aware that declining physical function is not necessarily an inevitable consequence of advancing kidney disease and that there is evidence that exercise training is associated with improved QoL [46-48]. There is also evidence that correction of anemia may improve QoL in CKD patients $[49,50]$. It seems reasonable to suggest that individually tailored exercise programs and correction of anemia should be considered for CKD patients who experience impaired functional status.

A limitation of the functional status and health-related QoL measures we used is their non-specificity for impairments related to advancing kidney disease. It is possible 
that individuals with stage 3 and 4 CKD experience early manifestations of symptoms common to more advanced kidney disease that are not captured by more generic QoL and functional status instruments [51]. Relevant to this possibility is a recent report by Agarwal [52] who surveyed the prevalence of symptoms in 92 individuals with stage 3 and 4 CKD commonly associated with impaired kidney function. He found that the most common symptoms of at least moderate severity included: 'tire easily', limited physical activity, nocturia, joint pain and 'stop and rest often'. Factor analysis identified four domains - neuropsychiatric, cardiovascular, uremia and anemia symptoms - that accounted for most (73\%) of the individual symptom variability. These observations, and our results, support the need to better understand the symptom burden of individuals with less advanced CKD and suggest that CKD-specific tools may be needed to effectively accomplish this task. We assessed the degree to which individuals excluded with missing creatinine values might have influenced our conclusions by assigning them to the group of individuals with eGFR $>90$ and re-running our adjusted analyses of mean scores. The results of this sensitivity analysis were comparable to those reported in the text and do not modify our conclusions. Finally, it is possible that other comorbidities associated with decreased kidney function that we did not assess during the baseline interview, like heart failure, periodic hypoglycemia, or obstructive sleep apnea, may have confounded our observations and additional studies are clearly indicated before we fully understand the relationship between functional status and QoL with decreased kidney function.

In conclusion, as kidney function decreases, individuals with CKD are increasingly likely to experience lower QoL, particularly physical limitations that cannot be attributed to other comorbid conditions. Clinicians caring for these individuals should be aware of the increased prevalence of impaired functional status and QoL, serially solicit information about the presence of these symp- toms as GFR declines, and be aware of the potential role that exercise programs and control of anemia may have in preventing or ameliorating this CKD-associated disease burden.

\section{Acknowledgments}

This research project is supported by a cooperative agreement U01 NS041588 from the National Institute of Neurological Disorders and Stroke, National Institutes of Health, Department of Health and Human Service. Additional funding was provided by an investigator-initiated grant-in-aid from Amgen Corporation. Amgen did not have any role in the design and conduct of the study, the collection, management, analysis, and interpretation of the data, or the preparation or approval of the manuscript. The manuscript was sent to Amgen for review prior to submission for publication. The content is solely the responsibility of the authors and does not necessarily represent the official views of the $\mathrm{Na}$ tional Institute of Neurological Disorders and Stroke or the National Institutes of Health. Representatives of the funding agency have been involved in the review of the manuscript but not directly involved in the collection, management, analysis or interpretation of the data. The authors acknowledge the participating investigators and institutions for their valuable contributions: The University of Alabama at Birmingham, Birmingham, Ala. (Study PI, Statistical and Data Coordinating Center, Survey Research Unit): George Howard, DrPH, Leslie McClure, PhD, Virginia Howard, $\mathrm{PhD}$, Libby Wagner, MA, Virginia Wadley, PhD, Rodney Go, PhD, Monika Safford, MD, Ella Temple, PhD, Margaret Stewart, MSPH, David Rhodes, RN; University of Vermont (Central Laboratory): Mary Cushman, MD; Wake Forest University (ECG Reading Center): Ron Prineas, MD, PhD; Alabama Neurological Institute (Stroke Validation Center, Medical Monitoring): Camilo Gomez, MD, Susana Bowling, MD; University of Arkansas for Medical Sciences (Survey Research): LeaVonne Pulley, PhD; University of Cincinnati (Clinical Neuroepidemiology): Brett Kissela, MD, Dawn Kleindorfer, MD; Examination Management Services, Incorporated (In-Person Visits): Andra Graham; Medical University of South Carolina (Migration Analysis Center): Daniel Lackland, DrPH; Indiana University (Neuropsychology Center): Frederick Unverzagt, PhD; National Institute of Neurological Disorders and Stroke, National Institutes of Health (funding agency): Claudia Moy, $\mathrm{PhD}$.

\section{References}

1 Kimmel PL, Patel SS: Quality of life in patients with chronic kidney disease: focus on end-stage renal disease treated with hemodialysis. Semin Nephrol 2006;26:68-79.

$\checkmark 2$ Kimmel PL, Peterson RA: Depression in end-stage renal disease patients treated with hemodialysis: tools, correlates, outcomes, and needs. Semin Dial 2005;18:91-97.

3 Kusek JW, Greene P, Wang SR, et al: Crosssectional study of health-related quality of life in African-Americans with chronic renal insufficiency: the African-American Study of Kidney Disease and Hypertension Trial. Am J Kidney Dis 2002;39:513-524.

-4 Rocco MV, Gassman JJ, Wang SR, Kaplan RM: Cross-sectional study of quality of life and symptoms in chronic renal disease patients: The Modification by Diet in Renal Disease Study. Am J Kidney Dis 1997;29: 888-896.
5 Cohen SD, Patel SS, Khetpal P, et al: Pain, sleep disturbance, and quality of life in patients with chronic kidney disease. Clin J Am Soc Nephrol 2007;2:919-925.

-6 Gorodetskaya I, Zenios S, McCulloch CE, et al: Health-related quality of life and estimates of utility in chronic kidney disease. Kidney Int 2005;68:2801-2808. 
7 Howard VJ, Cushman M, Pulley L, et al: The reasons for geographic and racial differences in stroke study: objectives and design. Neuroepidemiology 2005;25:135-143.

$\checkmark 8$ Radloff LS: The CES-D scale: a self-report depression scale for research in the general population. Appl Psychol Meas 1977;1:385-401.

-9 Melchior LA, Huba GJ, Brown VB, Reback CJ: A short depression index for women. Educ Psychol Meas 1993;53:1117-1125.

10 Hedayati SS, Bosworth HB, Kuchibhatla M, et al: The predictive value of self-report scales compared with physician diagnosis of depression in hemodialysis patients. Kidney Int 2006;69:1662-1668.

11 Irwin M, Artin KH, Oxman MN: Screening for depression in the older adult: criterion validity of the 10-Item Center for Epidemiologic Studies Depression Scale (CES-D). Arch Intern Med 1999;159:1701-1704.

-12 Cohen S, Kamarck T, Mermelstein R: A global measure of perceived stress. J Health Soc Behav 1983;24:385-396.

13 Cohen S, Williamson G: Perceived stress in a probability sample of the United States; in Spacapan S, Oskamp S (eds): The Social Psychology of Health: Claremont Symposium on Applied Social Psychology. Newbury Park/CA, Sage, 1988.

14 Ware JE, Kosinski M, Keller SD: A 12-Item Short-Form Health Survey: Construction of scales and preliminary tests of reliability and validity. Med Care 1996;34:220-233.

15 Idler EL, Benyamini Y: Self-rated health and mortality: a review of twenty-seven community studies. J Health Soc Behav 1997;38:21-37.

16 Idler EL, Russell LB, Davis D: Survival, functional limitations, and self-rated health in the NHANES I Epidemiologic Follow-up Study, 1992. First National Health and Nutrition Examination Survey. Am J Epidemiol 20001;152:874-883.

$\checkmark 17$ Levey AS, Stevens LA, Schmid CH, et al: CKD-EPI (Chronic Kidney Disease Epidemiology Collaboration): a new equation to estimate glomerular filtration rate. Ann Intern Med 2009;150:604-612.

18 Kleinbaum DG, Klein M: Logistic Regression - A Self-Learning Text, ed 2. New York, Springer, 2002.

19 SAS Institute Inc, Version 8. Cary, SAS Institute Inc, 2000.

20 Coresh J, Selvin E, Stevens LA, et al: Prevalence of chronic kidney disease in the United States. JAMA 2007;298:2038-2047.

-21 Harris LE, Luft FC, Rudy DW, Tierney WM: Clinical correlates of functional status in patients with chronic renal insufficiency. Am J Kidney Dis 1993;21:161-166.

22 Klang B, Bjorvell H, Clyne N: Quality of life in predialytic uremic patients. Qual Life Res 1996;5:109-116.

23 Shidler NR, Peterson RA, Kimmel PL: Quality of life and psychosocial relationships in patients with chronic renal insufficiency. Am J Kidney Dis 1998;32:557-566.
24 Korevaar JC, Jansen MA, Merkus MP, et al: Quality of life in predialysis end-stage renal disease patients at the initiation of dialysis therapy. The NECOSAD Study Group. Perit Dial Int 2000;20:69-75.

25 National Kidney Foundation: K/DOQI clinical practice guidelines for chronic kidney disease: evaluation, classification, and stratification. Kidney Disease Outcome Quality Initiative. Am J Kidney Dis 2002;39:S1S246.

26 Chow FY, Briganti EM, Kerr PG, et al: Health-related quality of life in Australian adults with renal insufficiency: a population-based study. Am J Kidney Dis 2003;41: 596-604.

27 Perlman RL, Finkelstein FO, Liu L, et al: Quality of life in chronic kidney disease (CKD): a cross-sectional analysis in the Renal Research Institute-CKD study. Am J Kidney Dis 2005;45:658-666.

28 Fukuhara S, Lopes AA, Bragg-Gresham JL, et al: Health-related quality of life among dialysis patients on three continents: the Dialysis Outcomes and Practice Patterns Study. Kidney Int 2003;64:1903-1910.

29 Mapes DL, Mapes DL, Lopes AA, et al: Health-related quality of life as a predictor of mortality and hospitalization: the Dialysis Outcomes and Practice Patterns Study. Kidney Int 2003;64:339-349.

30 Mapes DL, Bragg-Gresham JL, Bommer J, et al: Health-related quality of life in the Dialysis Outcomes and Practice Patterns Study (DOPPS). Am J Kidney Dis 2004;44:S54S60.

31 Lopes AA, Bragg-Gresham JL, Satayathum $S$, et al: Health-related quality of life and associated outcomes among hemodialysis patients of different ethnicities in the United States: the Dialysis Outcomes and Practice Patterns Study. Am J Kidney Dis 2003;41: 605-615.

- 32 Unruh M, Unruh M, Benz R, et al: Effects of hemodialysis dose and membrane flux on health-related quality of life in the HEMO study. Kidney Int 2004;66:355-366.

33 Unruh M, Miskulin D, Yan G, et al: Racial differences in health-related quality of life among hemodialysis patients. Kidney Int 2004;65:1482-1491.

34 Clase CM, Kiberd BA, Garg AX: Relationship between glomerular filtration rate and the prevalence of metabolic abnormalities: results from the Third National Health and $\mathrm{Nu}$ trition Examination Survey (NHANES III). Nephron Clin Pract 2007;105:c178-c184.

35 Gullion CM, Keith DS, Nichols GA, Smith DH: Impact of comorbidities on mortality in managed care patients with CKD. Am J Kidney Dis 2006; $48: 212-220$.

- 36 Keith DS, Nichols GA, Gullion CM, et al: Longitudinal follow-up and outcomes among a population with chronic kidney disease in a large managed care organization. Arch Intern Med 2004;164:659-663.
37 Hedayati SS, Jiang W, O’Connor CM, et al: The association between depression and chronic kidney disease and mortality among patients hospitalized with congestive heart failure. Am J Kidney Dis 2004;44:207-215.

-38 Odden MC, Whooley MA, Shlipak MG: Depression, stress, and quality of life in persons with chronic kidney disease: the Heart and Soul Study. Nephron Clin Pract 2006;103:c1c7.

39 Cohen SD, Norris L, Acquaviva K, et al: Screening, diagnosis, and treatment of depression in patients with end-stage renal disease. Clin J Am Soc Nephrol 2007;2:13321342.

-40 Cukor D, Peterson RA, Cohen SD, Kimmel PL: Depression in end-stage renal disease hemodialysis patients. Nat Clin Pract Nephrol 2006;2:678-687.

-41 Kimmel PL, Peterson RA, Weihs KL, et al: Psychosocial factors, behavioral compliance and survival in urban hemodialysis patients. Kidney Int 1998;54:245-254.

42 Lydick E, Epstein RS: Interpretation of quality of life changes. Qual Life Res 1993;2:221226.

43 Rubenach S, Shadbolt B, McCallum J, Nakamura T: Assessing health-related quality of life following myocardial infarction: is the SF-12 useful? J Clin Epidemiol 2002;55:306309.

44 Lim LL, Fisher JD: Use of the 12-Item ShortForm (SF-12) Health Survey in an Australian heart and stroke population. Qual Life Res 1999;8:1-8.

45 Clement FM, Klarenbach S, Tonelli M, et al: The impact of selecting a high hemoglobin target level on health-related quality of life for patients with chronic kidney disease: a systematic review and meta-analysis. Arch Intern Med 2009;169:1104-1112

-46 Fitts SS, Guthrie MR, Blagg CR: Exercise coaching and rehabilitation counseling improve quality of life for predialysis and dialysis patients. Nephron 1999;82:115-121.

47 Clyne N, Ekholm J, Jogestrand T, et al: Effects of exercise training in predialytic uremic patients. Nephron 1991;59:84-89.

48 Eidemak I, Haaber AB, Feldt-Rasmussen B, et al: Exercise training and the progression of chronic renal failure. Nephron 1997;75: $36-40$.

49 Strippoli GF, Navaneethan SD, Craig JC: Haemoglobin and haematocrit targets for the anaemia of chronic kidney disease. Cochrane Database Syst Rev 2006;4:CD003967.

50 Weisbord SD, Kimmel PL: Health-related quality of life in the era of erythropoietin. Hemodialysis Int 2008;12:6-15.

51 Murphy EL, Murtagh FE, Carey I, Sheerin NS: Understanding symptoms in patients with advanced chronic kidney disease managed without dialysis: use of a short patientcompleted assessment tool. Nephron Clin Pract 2009;111:c74-c80.

-52 Agarwal R: Developing a self-administered CKD symptom assessment instrument. Nephrol Dial Transplant 2010;25:160-166. 